\title{
Mucoepidermoid Carcinoma of the Eyelid -A Rare Case Report
}

\author{
Dr. Yapa Guha ${ }^{1}$, Dr Yumnam Chingsuingamba ${ }^{2}$ \\ ${ }^{1}$ (Department of Ophthalmology, Regional Institute of Medical Sciences, Imphal) \\ ${ }^{2}$ (Department of Ophthalmology, Regional Institute of Medical Sciences, Imphal)
}

\begin{abstract}
: we describe here a 43 year old woman who came to eye opd with a chief complaint of a mass in left lower eyelid since 4 years. On local examination the mass was non-tender, well defined margin, smooth, mobile and non-adherent to underlying tarsal plate. Other ocular examinations were normal. General investigations were done. The mass was excised by simple excision under peribulbar local anaesthesia and sent for histopathological examination. The report showed a well encapsulated malignant tumour composed of intermediate cells in sheets admixed with spindloid cells. Many polygonal cells with oncocytic cytoplasm were also seen in the sheets. Abnormal mitosis was noted. The features were suggestive of mucoepidermoid carcinoma- spindloid oncocytic variant. Mucoepidermoid carcinoma of eyelid is a rare malignant sweat gland neoplasm. To our knowledge this is the $1^{\text {st }}$ report of mucoepidermoid carcinoma of eyelid here in our Institute.

Keywords: eyelid tumour, eyelid mass, mucoepidermoid carcinoma
\end{abstract}

\section{INTRODUCTION}

A 43 year old woman came to eye opd with a chief complaint of a mass in left lower eyelid since 4 years. The mass to begin with was small in size but gradually increased in size. It was painless with no history of trauma. She did not have any other complaint beside the mass. No similar history in the past. Family history and personal history were not significant.

\section{OPHTHALMOLOGIC EXAMINATION}

Ophthalmological examinations included the vision, local examination of the eye using torch illumination, slitlamp and fundus ophthalmoscopy, and intraocular pressure measurement. Emphasis was made on local examination of the eyelid mass. Following were the findings in regard to local examination the eyelid mass.

1. Inspection: $5 \mathrm{~mm}$ below the lateral canthus, $7 \mathrm{mmx} 7 \mathrm{~mm}$ size, smooth surface with slight palor

2. Palpation: non-tender, firm, well defined margin, mobile and non adherent to underlying tarsal plate

Anterior segment was examined by torch illumination and slit lamp. Conjunctiva, cornea, anterior chamber, iris and pupil were all within normal limit. Posterior segment and Fundus examination was done using direct and indirect ophthalmoscope. It was normal in both the eyes. Vision of both eyes were $6 / 6 \mathrm{p}$ ' by snellens chart. Intraocular pressure was 14 in both the eyes by goldmann applanation tonometer.

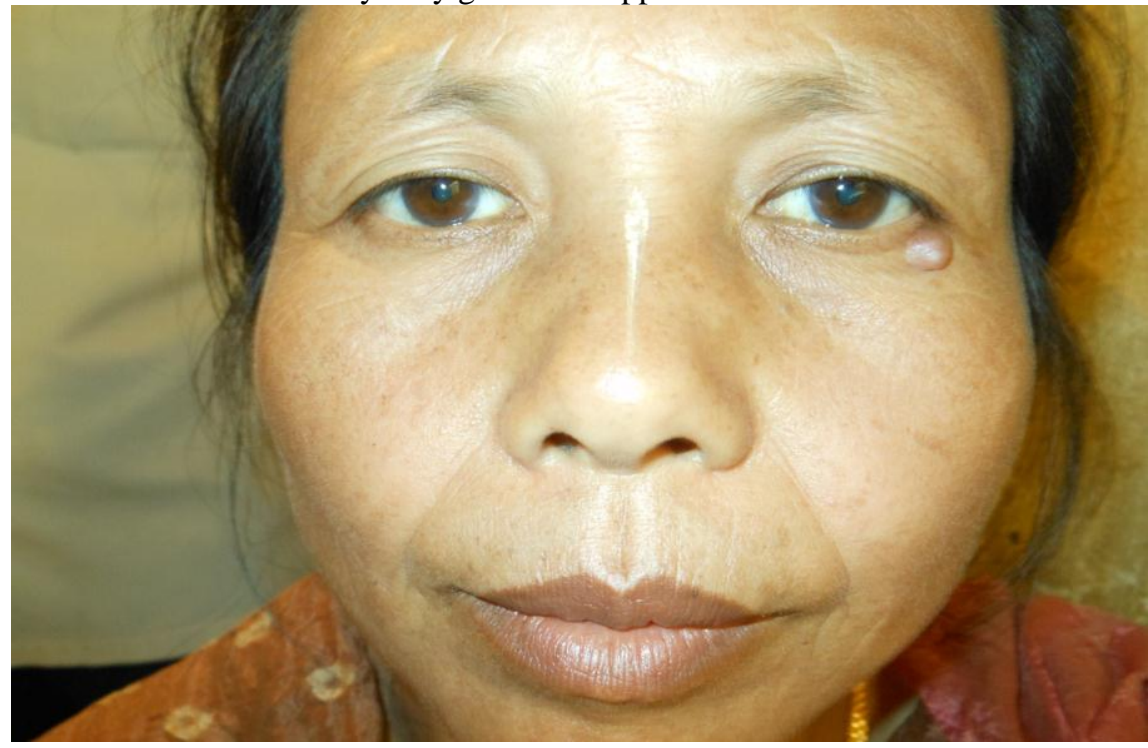

Fig. 1 (Pre-op) 


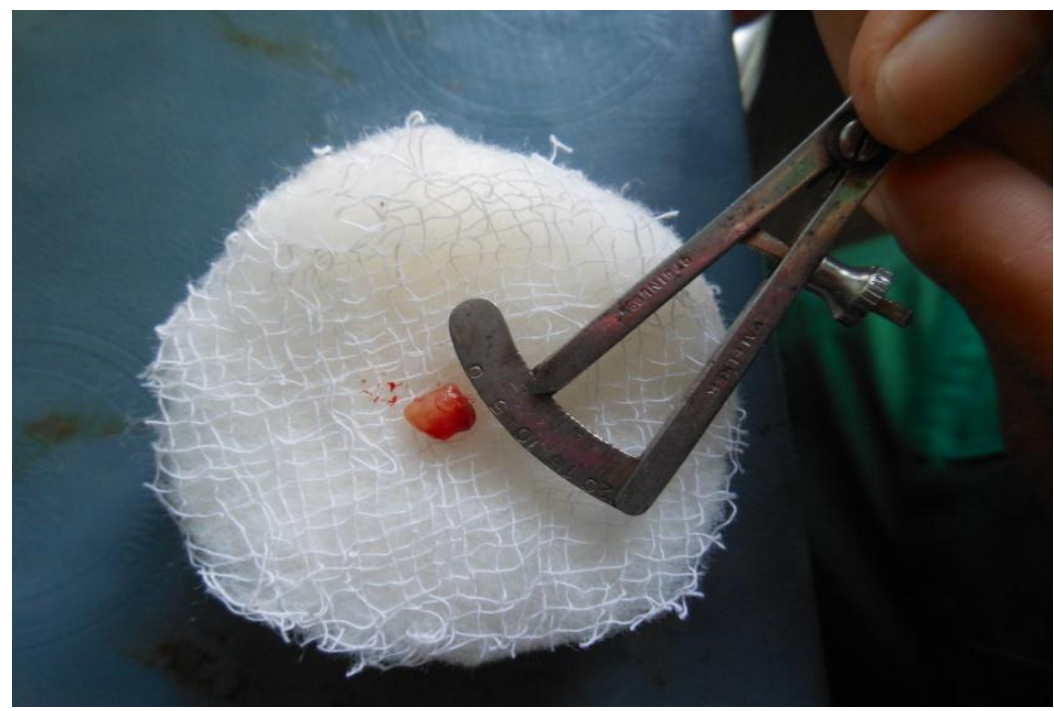

Fig. 2 (Excised mass)

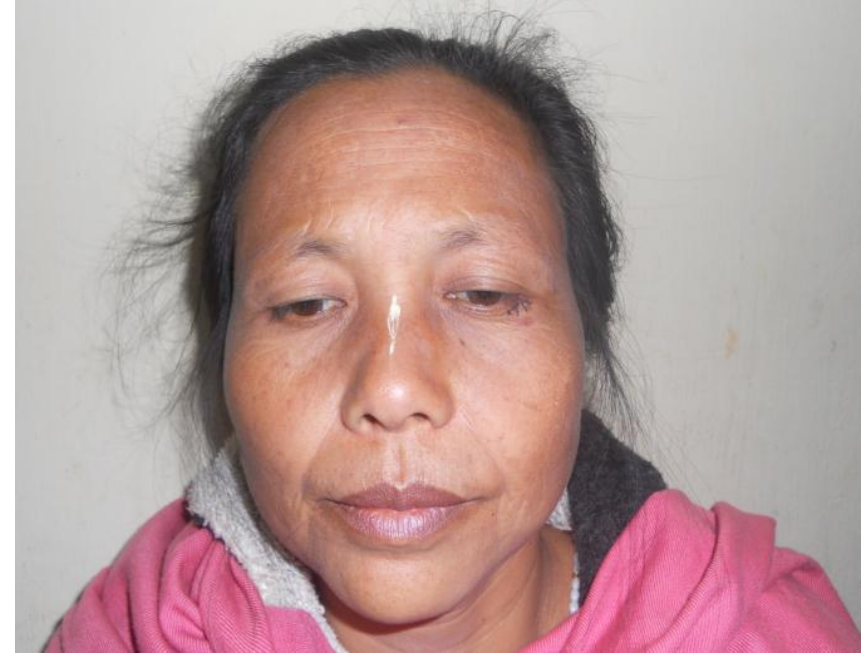

Fig. 3 (post-op)

\section{III.}

MANAGEMENT

General investigations were done. The patient was referred for medical clearance. The mass was then excised under peribulbar local anaesthesia by simple excision. It measured $5 \mathrm{~mm} \times 5 \mathrm{~mm}$ using a caliper. The excised mass was then sent for histopathological examination. The report revealed mucoepidermoid carcinoma of eyelid. Following features were noted in the mass pathology:

1. Well encapsulated malignant tumour composed of intermediate cells in sheets admixed with spindloid cells with large round to oval cells with vesicular nuclei and distinct nucleoli

2. Polygonal cells with oncocytic cytoplasm also seen in the sheets

3. Many foci with goblet cells with mucin

4. Abnormal mitosis

Thus post-op diagnosis of mucoepidermoid carcinoma of eyelid was made.

IV.

\section{DISCUSSION}

Mucoepidermoid carcinoma of eyelid is a rare malignant sweat gland neoplasm. ${ }^{1-3}$ The clinial diagnosis from the other common tumours in the eyelid area may be difficult because this tumour does not have a pathognomonic clinical appearance and is always asymptomatic. It has higher degree of malignancy than the basal cell ca and squamous cell ca. ${ }^{4}$ it also displays an unusual capacity of aggressive local invasion and recurs easily after simple excision. Sometimes may require enucleation or exenteration when orbit is involved. Histologically the tumour exhibits an admixture of epidermoid cells and mucous secreting cells in variable proportions. The cytoplasm of the cells usually appears clear or vacuolated, sometimes like a signet ring. The histochemical stain for mucin (periodic acid schiff, alcian blue, mucicarmine) can confirm the diagnosis. The other cells which can be present are intermediate cells, basal cells and more rarely, hydropic cells and oncocytic 
cells. Frozen section diagnosis of this tumour can be difficult due to the rarity of its occurrence. Management recommended is surgical excision with frozen section control by Mohs' technique. The prognosis for life in mucoepidermoid carcinoma is reported to be good. ${ }^{5}$

\section{CONCLUSION}

Mucoepedermoid carcinoma of eye is rare. Tumour of ocular adnexa had high recurrence rate with aggressive local invasion. So, early diagnosis and management with extended follow up is required in such cases. Clinically may be indistinguishable from sq cell ca or basal cell ca but a histological examination of the tumour would establish the diagnosis. Management recommended is surgical by wide local excision by Moh's technique.

\section{REFERENCES}

[1] Brownstein S. Mucoepidermoid carcinoma of the conjunctiva with intraocular invasion. Ophthalmology 88:1226-1230, 1981

[2] Bambirra EA, Miranda D, Rayes A. Mucoepidermoid tumour of the lacrimal sac. Arch Ophthalmol 99:2149-2150, 1981

[3] Ni C, Wagoner MD, Wang WJ, Albert DM, Fan CO, Robinson N. Mucoepidermoid carcinoma of the lacrimal sac. Arch Ophthalmol 101:1572-1574, 1983

[4] Zhang h, Yan J, Li Y, Zhang P. mucoepidermoid ca of the eyelid;a case report and review of literature.yan ke xue bao 2005 sep:21(3):152-7

[5] Rao NA, and Font RL. Mucoepidermoid carcinoma of the conjunctiva, a clinicopathologic study of five cases. Cancer 38:1699-1709, 1976 